Extended abstract for the 20th AIAA Applied Aerodynamics Conference

\title{
Parametric Study of a YAV-8B Harrier in Ground Effect Using Time-Dependent Navier-Stokes Computations
}

\author{
Shishir Pandya ${ }^{¥}$ and Neal Chaderjian ${ }^{*}$ \\ NASA Ames Research Center, Moffett Field, CA 94035 \\ and \\ Jasim Ahmad ${ }^{\#}$ \\ ELORET, Moffett Field, CA 94035
}

\section{Introduction}

Flow simulations using the time-dependent Navier-Stokes equations remain a challenge for several reasons. Principal among them are the difficulty to accurately model complex flows, and the time needed to perform the computations. A parametric study of such complex problems is not considered practical due to the large cost associated with computing many time-dependent solutions. The computation time for each solution must be reduced in order to make a parametric study possible. With successful reduction of computation time, the issue of accuracy, and appropriateness of turbulence models will become more tractable.

A parametric study to generate a longitudinal stability and control database on the YAV-8B Harrier aircraft in near-hover conditions has been discussed in a previous paper[1 ][2 ]. Two main issues with computing Harrier flow fields are complex geometry and complex flow physics. The Chimera overset grid approach is used to address the geometric complexity. An overset mesh with 67 zones is generated around the YAV-8B to compute the flow field in the vicinity of the aircraft and the ground plane. The flow field with two high-speed rear jets and two front jets impacting the ground from an aircraft fixed at heights between 10 and 30 feet, is complex and time-varying. At each height, computations are carried out at several angles of attack to generate a database of force coefficients. The OVERFLOW code[3 ] is used to compute the time dependent, viscous flows. Each computation for the generation of the database is costly because it involves long run times to simulate unsteady flow with low dominant frequencies $(\sim 1 \mathrm{~Hz})$. In a first attempt to generate a database, it took more than one month to generate one solution, without taking grid generation into account. The bottlenecks included the slow decay of numerical transients associated with the solution start-up process, and the lack of scalability to more than 8 processors.

In our previous paper [1], the emphasis is put on reduction of the time to generate a solution. The computations in the earlier paper were done using a first order accurate method in time. Small time steps had to be used in order to maintain algorithm stability to capture the low-speed ambient

\footnotetext{
₹esearch Scientist, Member AIAA

${ }^{*}$ Research Scientist,Associate Fellow AIAA

\# Senior Research Scientist
} 